\title{
Conjugation of a Blood Brain Barrier Peptide Shuttle to an Fc domain for brain delivery of therapeutic biomolecules
}

\author{
Marco Cavaco ${ }^{\ddagger \dagger}$, Silvia Frutos ${ }^{\S}$, Paula Oliete ${ }^{\S}$, Javier Valle ${ }^{\dagger}$, David Andreu ${ }^{\dagger}$, Miguel A. R. B. \\ Castanho ${ }^{\ddagger}$, Miquel Vila-Perelló ${ }^{\S, *}$, and Vera Neves ${ }^{\ddagger}, *$ \\ Instituto de Medicina Molecular João Lobo Antunes, Faculdade de Medicina, Universidade de Lisboa, Av. \\ Prof Egas Moniz, 1649 - 028 Lisboa, Portugal; \\ 'Proteomics and Protein Chemistry Unit, Department of Experimental and Health Sciences, Pompeu Fabra \\ University, Dr. Aiguader 88, Barcelona Biomedical Research Park, 08003 Barcelona, Spain; \\ §SpliceBio S.L., Baldiri Reixac 10 - 12, 08028 Barcelona, Spain;
}

\begin{abstract}
The frequency of brain disease has increased significantly in the past years. After diagnosis, therapeutic options are usually limited, which demands the development of innovative therapeutic strategies. The use of antibody-drug conjugates (ADCs) is promising but highly limited by the existence of the blood-brain barrier (BBB). To overcome the impermeability of this barrier, antibody fragments can be engineered and conjugated to BBB peptide shuttles (BBBpS), which are capable of brain penetration. Herein, we linked the highly efficient BBBpS, $\mathrm{PepH} 3$, to the IgG fragment crystallizable (Fc) domain using streamlined expressed protein ligation (SEPL) method. With this strategy, we obtained an Fc-PepH3 scaffold that can carry different payloads. Fc-PepH3 was shown to be non-toxic, capable of crossing an in vitro cellular BBB model, and binding to neonatal $\mathrm{Fc}$ receptor ( $\mathrm{FcRn}$ ), which is responsible for antibodies long half-life $\left(\mathrm{t}_{1 / 2}\right)$. Overall, we demonstrated the potential of $\mathrm{Fc}-\mathrm{PepH} 3$ as a versatile platform readily adaptable to diverse drugs of therapeutic value to treat different brain conditions.
\end{abstract}

KEYWORDS: Antibody fragments; BBB peptide shuttle; brain disorders; site-specific conjugation; streamlined expressed protein ligation 


\section{Table of Content}

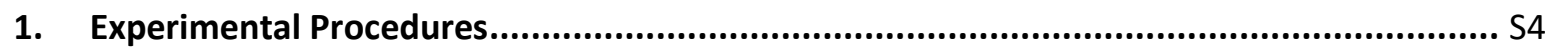

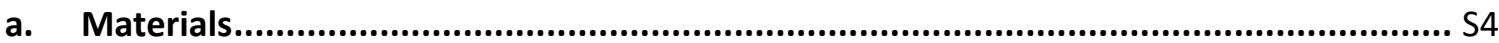

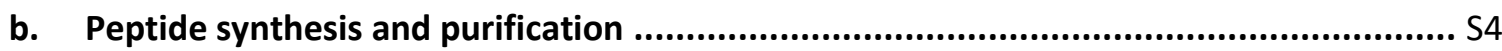

Figure S1 | RP-HPLC and MS analysis of the purified Cys-PepH3. (A) RP-HPLC of purified CysPepH3 over a $10-50 \%$ B gradient on a Luca C18 column (220 nm detection). (B) MS of the entire HPLC peak. The presence of a species with a MW in good agreement with the Cys-PepH3 peptide. No peaks corresponding to impurities were detected ( $\left.\mathrm{MW}_{\text {calc: }}: 1,059.3 \mathrm{Da}\right) . \quad$ S5

Table S1. Peptides synthetized. S6

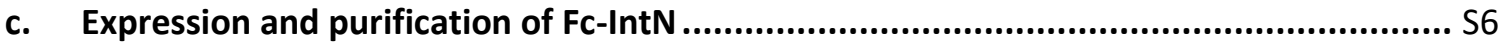

d. Thiolysis/ligation of Cys-PepH3 to the Fc-IntN .......................................................... S6

e. Characterization of Fc-PepH3 conjugate ...................................................................... S6

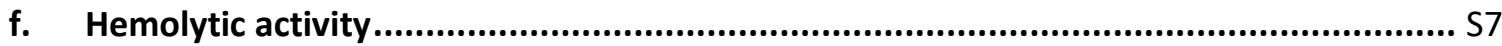

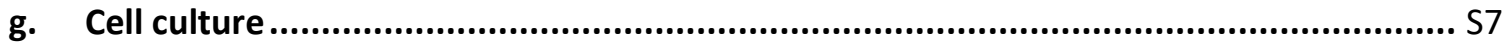

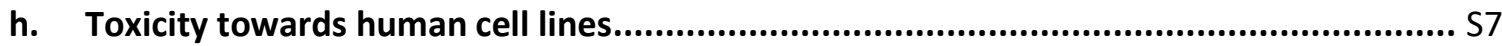

i. Translocation across a human endothelial cell line ................................................. S8

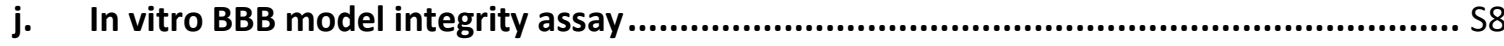

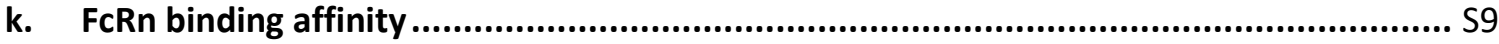

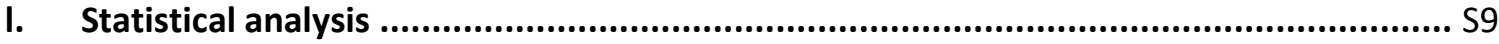

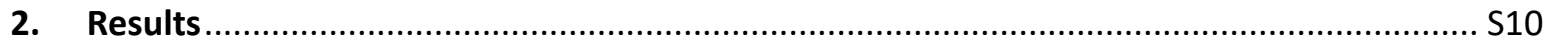

Figure S2. in vitro toxicity of antibody fragments. $\mathrm{HBEC}-5 \mathrm{i}, \mathrm{Hs} 68$, and $\mathrm{RBCs}$ were incubated with $100 \mu \mathrm{L}$ of previously diluted antibody fragments $(0.001-100.0 \mu \mathrm{M}$ range) for $24 \mathrm{~h}$. The values were obtained from triplicates of three independent experiments. Error bars, S.D. S10

Figure S3. Translocation of antibody fragments across an in vitro BBB model and the FD40 permeability study. Percentage of translocation of antibody fragments (25 ng) and fluorescence intensity of FD40 measured after translocation assay. The values were obtained from triplicates of three independent experiments. Error bars, S.D.

S11

Figure S4. Binding of proteins to the FcRn. Antibody fragments' binding curves to immobilized FcRn at pH 6.0, obtained using a single-site fit model. The values were obtained from triplicates of three different experiments. Error bars, S.D. $\quad \mathrm{S} 12$

3. Supplementary data - in vitro HBEC-5i characterization ............................................... S13 


\section{SUPPORTING INFORMATION}

Figure S5. Confocal images of tight junction proteins in HBEC-5i cells. (A) Control, (B) Claudin5, (C) Occludin-1, and (D) ZO-1. Images were obtained using a 40x water-objective. Nuclei stained with Hoeschst 33342 (blue) and tight junction proteins (red). Bar represents $10 \mu \mathrm{m} .13$

Figure S6. Transport of Lucifer Yellow (LY) across HBEC-5i monolayers. Ly applied at a concentration of $50.0 \mu \mathrm{M}$ in serum-free medium. HBEC-5i monolayers were incubated with LY in or without the presence of EGTA. Experiments were performed in triplicates on different days using independently grown cell cultures. Data is shown as mean \pm SD.

Figure S7. Transport of human Transferrin (hTf) across HBEC-5i monolayers. hTf applied at a concentration of $400.0 \mathrm{\mu g} / \mathrm{mL}$ in serum-free medium. HBEC-5i monolayers were incubated with $\mathrm{hTf}(24 \mathrm{~h})$ in or without the presence of EGTA. Experiments were performed in triplicates on different days using independently grown cell cultures. Data is shown as mean $\pm S D$

Figure S8. Confocal images of HBEC-5i cells incubated for 20 min at $37^{\circ} \mathrm{C}$ with Human Transferrin at a concentration of $25 \mu \mathrm{g} / \mathrm{mL}$ in HEPES supplemented with $1 \mathrm{M}$ glucose, $0.2 \mathrm{M}$ $\mathrm{MgCl}_{2}$, and 1\% BSA. (A - B) 20x air-objective, (C - D) 63x oil-objective. Nuclei stained with Hoeschst 33342 (blue) and transferrin Alexa Fluor 568 conjugate (red). Bar represents $10 \mu \mathrm{m}$. 


\section{SUPPORTING INFORMATION}

\section{Experimental Procedures}

\section{a. Materials}

Fmoc-protected amino acids, Fmoc-Rink amide (MBHA) resin, 2-(1H-benzotriazol-1-yl)-1, 1,3,3tetramethyluronium hexafluorophosphate (HBTU), and N-hydroxybenzotriazole (HOBt) were from Iris Biotech (Marktredwitz, Germany). HPLC-grade acetonitrile (ACN), and peptide-synthesis grade $\mathrm{N}, \mathrm{N}$-dimethylformamide (DMF), dichloromethane (DCM), N,N-diisopropylethylamine (DIEA), N,Ndiisopropylcarbodiimide (DIPCI), trifluoroacetic acid (TFA), and triisopropylsilane (TIS) were from Carlo Erba-SDS (Sa-badell, Spain). 3,6-dioxa-1,8-octanedithiol (DODT), and fluorescein isothiocyanate-40 KDa dextran (FD40) were from Sigma-Aldrich (Madrid, Spain).

Opti-minimal essential medium (Opti-MEM ${ }^{\circledR}$ ), Dulbecco's Modified Eagle Medium (DMEM), DMEM/Ham's F-12 (DMEM:F12), DMEM:F12 without phenol-red, trypsin-EDTA, attachment factor protein solution (AF), fetal bovine serum (FBS), penicillin-streptomycin antibiotic solution (Pen/Strep), and Expi293 Expression System were from Gibco/Thermo Fischer (Gaithersburg, Maryland, USA). Endothelial cell growth supplement (ECGS) was from Sigma-Aldrich (Madrid, Spain). CellTiter-Blue ${ }^{\circledR}$ Cell Viability Reagent was from Promega (Madrid, Spain).

All buffering salts, isopropyl- $\beta$-D-thiogalactopyranoside (IPTG), Tris(2-carboxyethyl)phosphine hydrochloride (TCEP), and N,N-diisopropylethylamine (DIPEA) were from Thermo Fisher (Gaithersburg, Maryland, USA). Sodium 2-mercaptoethanesulfonate (MES), ethanedithiol (EDT), and Coomassie brilliant blue were from Sigma-Aldrich (Madrid, Spain). Complete protease inhibitor tables were from Roche Diagnostics (Mannheim, Germany). Criterion XT Bis-Tris gels (12\%), Immuno-blot PVDF membrane $(0.2 \mu \mathrm{m})$, Immuno-blot nitrocellulose membrane, Bradford reagent dye concentrate, Tris/Glycine/SDS running buffer, Tris/Glycine transfer buffer were from Bio-Rad (Hercules, California, USA). ECL Prime western blotting detection reagent from GE Healthcare (Chicago, Illinois, USA). Goat anti-human IgG-HRP from Abcam (Cambridge, UK).

\section{b. Peptide synthesis and purification}

Cys-PepH3 (C(Ahx)AGILKRW-amide) was synthetized in a Prelude Synthesizer (Gyros Protein Technologies, USA) running Fmoc (FastMoc) SPPS protocols at $0.1 \mathrm{mmol}$ scale on a Fmoc-Rink-amide ChemMatrix resin (Table S1). Side chain functionalities were protected with NG-2,2,4,6,7pentamethyldihydrobenzofuran-5-sulfonyl (Arg), Na-tert-butoxycarbonyl (Trp), and trityl (Cys) groups. Eight-fold excess of Fmoc-L-amino acids and HBTU, in the presence of a double molar amount of DIEA, were used for the coupling steps, with DMF as solvent. After chain assembly, full depro-tection and cleavage were carried out with TFA/H2O/DODT/TIS (94:2.5:2.5:1, v/v, $90 \mathrm{~min}$, rt). Peptides were isolated by precipitation with cold diethyl ether and centrifugation at $4,000 \mathrm{xg}, 4^{\circ} \mathrm{C}$ for 20 min. Then, they were taken up in $\mathrm{H} 2 \mathrm{O}$ and lyophilized.

Analytical reversed-phase (RP) HPLC was performed on a Luna C18 column $(4.6 \times 50 \mathrm{~mm}, 3 \mu \mathrm{m}$; Phenomenex, Tor-rance, California, USA) using linear gradients of solvent B (0.036\% TFA in MeCN) into solvent $A(0.045 \%$ TFA in $\mathrm{H} 2 \mathrm{O})$ at a flow rate of $1 \mathrm{~mL} / \mathrm{min}$ and with UV detection at $220 \mathrm{~nm}$. Preparative RP-HPLC was performed on a Luna C18 column $(21.2 \times 250 \mathrm{~mm}, 10 \mu \mathrm{m}$; Phenomenex, Torrance, Cali-fornia, USA) using linear gradients of solvent $B(0.1 \%$ TFA in MeCN) into solvent $A$ 


\section{SUPPORTING INFORMATION}

(0.1\% TFA in $\mathrm{H} 2 \mathrm{O}$ ) with a flow rate of $25 \mathrm{~mL} / \mathrm{min}$ and with UV detection at $220 \mathrm{~nm}$ (Figure S1A). LCMS was performed in a LC-MS 2010EV instru-ment (Shimadzu, Kyoto, Japan) fitted with an XBridge C18 column ( $4.6 \times 150 \mathrm{~mm}, 3.5 \mu \mathrm{m}$; Waters, Spain), eluting with linear gradients of $\mathrm{HCOOH} / \mathrm{MeCN}$ $(0.08 \% \mathrm{v} / \mathrm{v})$ into $\mathrm{HCOOH} / \mathrm{H} 2 \mathrm{O}(0.1 \% \mathrm{v} / \mathrm{v})$ over $15 \mathrm{~min}$ at $1 \mathrm{~mL} / \mathrm{min}$ (Figure S1B). Peptide stock solutions $(1 \mathrm{mM})$ in filtered $\mathrm{H} 2 \mathrm{O}$ were stored at $-20^{\circ} \mathrm{C}$.

A

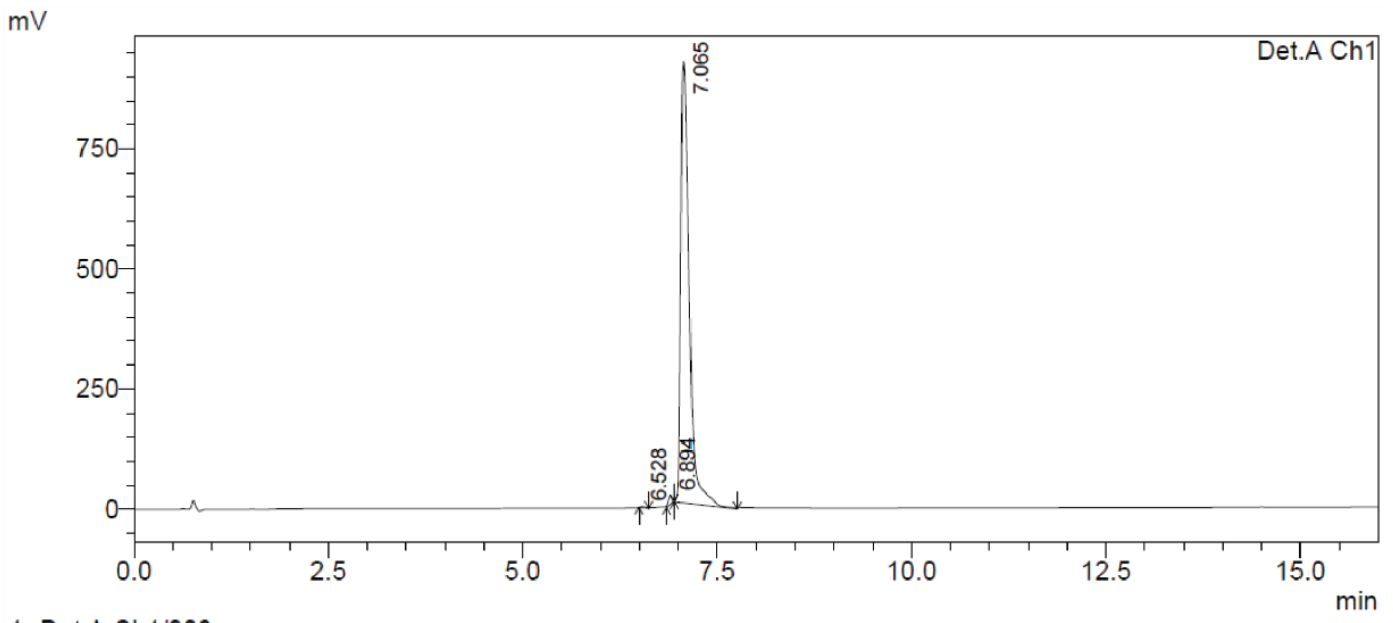

1 Det.A Ch1/220nm

PeakTable

Detector A Ch1 220nm
\begin{tabular}{|r|r|r|r|r|r|}
\hline Peak\# & Ret. Time & \multicolumn{1}{|c|}{ Area } & \multicolumn{1}{c|}{ Height } & Area $\%$ & \multicolumn{1}{c|}{ Height $\%$} \\
\hline 1 & 6.528 & 15958 & 3051 & 0.230 & 0.324 \\
\hline 2 & 6.894 & 64531 & 18859 & 0.929 & 2.005 \\
\hline 3 & 7.065 & 6868959 & 918652 & 98.842 & 97.671 \\
\hline Total & & 6949448 & 940562 & 100.000 & 100.000 \\
\hline
\end{tabular}

B

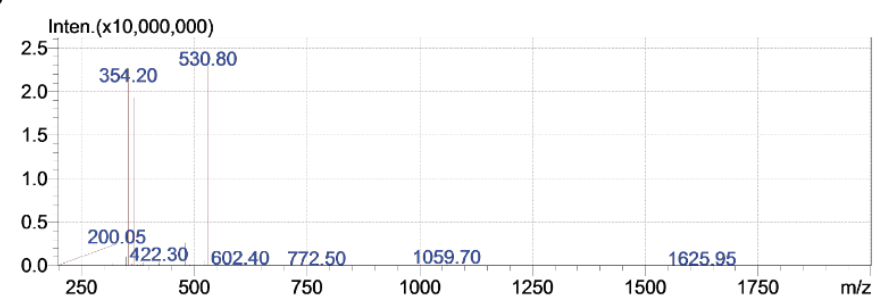

Figure S1 | RP-HPLC and MS analysis of the purified Cys-PepH3. (A) RP-HPLC of purified Cys-PepH3 over a 10 $-50 \%$ B gradient on a Luca C18 column (220 nm detection). (B) MS of the entire HPLC peak. The presence of a species with a $\mathrm{MW}$ in good agreement with the Cys-PepH3 peptide. No peaks corresponding to impurities were detected (MW $\mathrm{Malc}_{\text {: }}$ 1,059.3 Da). 


\section{SUPPORTING INFORMATION}

Table S1. Peptides synthetized.

\begin{tabular}{|c|c|c|c|c|c|}
\hline Peptide & Amino Acid Sequence & Theoretical mass $(\mathrm{Da})^{\mathbf{a}}$ & Experimental mass $(D a)^{b}$ & $\begin{array}{c}\mathbf{H P L C ~ t}_{\mathbf{R}} \\
(\mathrm{min})\end{array}$ & $\begin{array}{c}\text { Purity } \\
(\%)^{\mathrm{c}}\end{array}$ \\
\hline C-PepH3 & C(Ahx)AGILKRW-amide & 1059.3 & 1060.6 & 7.065 & 97.7 \\
\hline
\end{tabular}

\section{c. Expression and purification of Fc-IntN}

Expi293 cells were transiently transfected with Fc-IntN constructs using Expi293 Expression System, according to the manufacturer's instructions. Briefly, cells were grown in suspension in Expi293 Expression Medium, in vented-cap shaker flasks for 5 days at $37^{\circ} \mathrm{C}$. Prior to the day of transfection, Expi293 cells were seeded at $2.5 \times 106$ cells $/ \mathrm{mL}$. The day of transfection, Expi293 cells were seeded at $3.0 \times 10^{6}$ cells $/ \mathrm{mL}$. Transfection complexes were prepared by mixing $80 \mu \mathrm{l}$ of ExpiFectamine 293 transfection reagent with $30 \mu \mathrm{g}$ of plas-mid DNA in $1.5 \mathrm{~mL}$ Opti-MEM ${ }^{\circledR}$ medium. After 20 min incubation at r.t., transfection mixtures were added to a suspension of Expi293 cells in $30 \mathrm{~mL}$ volume. After $24 \mathrm{~h}, 150 \mu \mathrm{L}$ of ExpiFectamine transfection Enhancer 1 and $1.5 \mathrm{~mL}$ of ExpiFectamine Transfection Enhancer 2 were added to the transfected cells.

After 5 days incubation, cell supernatant was harvested and spun down at 2,000 xg for 20 min at $4^{\circ} \mathrm{C}$ and filtered through a $0.22 \mathrm{~mm}$ filter to remove any cell debris. After addition of Complete Protease Inhibitors, cell supernatant was directly purified over the Ni-beads using the standard protocol, after which the eluted Fc-IntN was dialyzed into 1x PBS (137 mM NaCl, $2.7 \mathrm{mM} \mathrm{KCl}, 10 \mathrm{mM}$ $\mathrm{Na} 2 \mathrm{HPO} 4$, and $1.8 \mathrm{mM} \mathrm{KH2PO}$, pH 7.4) and 1 mM EDTA.

\section{d. Thiolysis/ligation of Cys-PepH3 to the Fc-IntN}

Ni-beads purified Fc-IntN at $500 \mu \mathrm{g} / \mathrm{mL}$ was mixed with $25 \mu \mathrm{M}$ of IntC and $0.5 \mathrm{mM}$ Cys-PepH3 in 100 $\mathrm{mM}$ phosphate, $150 \mathrm{mM} \mathrm{NaCl}, 1 \mathrm{mM}$ EDTA, $100 \mathrm{mM}$ MESNa, $1 \mathrm{mM}$ TCEP, pH 7.2. Reaction was allowed to proceed, protected from light at $r$.t. for $24 \mathrm{~h}$. The reaction endpoint was monitored by SDS-PAGE, then the reaction mixture was extensively dialyzed into $1 \times$ PBS to remove excess peptide and MESNa. The Fc-PepH3 conjugate was then purified over a Superdex S200 column on an Akta purification system (GE Healthcare; Chicago, Illinois, USA).

\section{e. Characterization of Fc-PepH3 conjugate}

Fc-PepH3 conjugate was analyzed by RP-HPLC/MS and size-exclusion chromatography. 50 $\mu \mathrm{g}$ of FcPepH3 was used for RP-HPLC/MS analysis. Before the analysis, the sample was deglycosylated and fully reduced. Deglycosylation was performed using $2 \mathrm{U}$ of PNGase F per $\mu \mathrm{g}$ of sample and incu-bated at $37^{\circ} \mathrm{C}$ o.n, then denatured by exchanging sample buffer to $6 \mathrm{M} \mathrm{Gn}-\mathrm{HCl}, 100 \mathrm{mM}$ phosphate, 150 $\mathrm{mM} \mathrm{NaCl}, 1 \mathrm{mM}$ EDTA at $\mathrm{pH} 7.2$ and fully reduced by treatment with $10 \mathrm{mM} \mathrm{DTT}$ at $37^{\circ} \mathrm{C}$ for $1 \mathrm{~h}$. The analysis was performed on a Zorbax 300SB C8 column using a 15-70\% linear gradient of solvent B into solvent $A$ over $30 \mathrm{~min}$ at $1 \mathrm{~mL} / \mathrm{min}$ flow rate and $70^{\circ} \mathrm{C}$, preceded by $3 \mathrm{~min}$ isocratic elution at 


\section{SUPPORTING INFORMATION}

$15 \%$ B. Sol-vent A was $0.02 \%$ TFA and $0.25 \%$ FA (formic acid) in $\mathrm{H} 2 \mathrm{O}$. Solvent B was $90 \%$ isopropanol with $0.02 \%$ TFA and $0.25 \%$ FA in H2O. HPLC peaks were collected and analyzed by MS.

The integrity of Fc-PepH3 conjugate was confirmed by in-jecting $50 \mu \mathrm{g}$ of ligated and dialyzed conjugate in running buffer (100 mM phosphate, $150 \mathrm{mM} \mathrm{NaCl}, 1$ mM EDTA, 1 mM TCEP, pH 7.2) into an S200 size-exclusion column in the Akta purification system.

\section{f. Hemolytic activity}

Fresh human blood was collected in EDTA tubes and centrifuged at $1,000 \times \mathrm{gg}$ for $10 \mathrm{~min}$ at $4^{\circ} \mathrm{C}$. The supernatant was discharged, and the pellet containing RBCs was washed three times with 1x PBS and resuspended in $1 \times$ PBS to obtain a $2.0 \%(\mathrm{v} / \mathrm{v})$ suspension. Then, RBCs were added to centrifuge tubes containing 2-fold serially diluted conjugates to a final concentration ranging from 0.001 to 100 $\mu \mathrm{M}$. The suspension was incubated for $24 \mathrm{~h}$ at $37^{\circ} \mathrm{C}$ with gentle stirring. After that, samples were centrifuged for $2 \mathrm{~min}$ at $1,000 \mathrm{xg}$. Supernatants were transferred to 96 -well plates, and the hemoglobin released measured by absorbance at $570 \mathrm{~nm}$ in a Varioskan ${ }^{\mathrm{TM}}$ LUX multimode microplate reader (Thermo Fisher, Madrid, Spain). 1x PBS with no peptides and Triton X-100 at 1\% and $4 \%(\mathrm{v} / \mathrm{v})$ were used as negative and positive controls, respectively. Hemolytic activity (\%) was determined using the following equation:

$$
\text { Hemolysis }(\%)=\left(\frac{A b s_{P T}-A b s_{N C}}{A b s_{P C}-A b s_{N C}}\right) \times 100
$$

$A b S_{P T}$ is the absorbance of treated samples, $A b s_{N C}$ is the absorbance from negative control, and $A b S_{P C}$ absorbance from positive control.

$\mathrm{HC}_{50}$ values were determined using the GraphPad Prism 7.0 software using a log(inhibitor) vs. normalized response. Experiments were performed on different days using independent blood donors.

\section{g. Cell culture}

Adherent human brain endothelial cells (HBEC-5i, ATCC ${ }^{\circledR}$ CRL-3345TM) were grown in AF-coated Tflasks as monolayer in DMEM:F12 supplemented with 10\% FBS, $1 \%$ pen-strep, and $15 \mu \mathrm{g} / \mathrm{mL}$ ECGS. Adherent human fibroblasts cells (Hs68, ATCC $^{\circledR}$ CRL-1635TM) were grown as monolayer in DMEM supplemented with $10 \%$ FBS, and $1 \%$ pen-strep. Cells were cultured in $95 \%$ humidified atmosphere and $5 \% \mathrm{CO} 2$ at $37{ }^{\circ} \mathrm{C}$ (MCO-19AIC (UV), Sanyo, Japan), with the medium changed every other day.

\section{h. Toxicity towards human cell lines}

Protein's cytotoxicity towards HBEC-5i and Hs68 cell lines was determined using the CellTiter-Blue ${ }^{\circledR}$ cell viability assay, following a described protocol. Briefly, HBEC-5i and Hs68 were carefully harvested with trypsin-EDTA and seeded at 15,000 cells/100 $\mu \mathrm{L}$ into 96 -well clear flat-bottomed polystyrene plates (Corning, New York, USA) for $24 \mathrm{~h}$. After medium removal, cells were washed two 


\section{SUPPORTING INFORMATION}

times with $1 x$ PBS, and $100 \mu \mathrm{L}$ of antibody fragments $(0.001-100 \mu \mathrm{M}$ range $)$ in the respective medium were added to cells. After $24 \mathrm{~h}$, cells were washed two times with $1 \mathrm{x}$ PBS and $20 \mu \mathrm{L}$ of CellTiter-Blue ${ }^{\circledR}$ reagent (diluted in $100 \mu \mathrm{L}$ medium) was added to each well and incubated for $3 \mathrm{~h}$ in culturing conditions. The fluorescence intensity was measured using VarioskanTM LUX multimode microplate reader (Thermo Fisher, Madrid, Spain).

$I C_{50}$ values were determined using GraphPad Prism 7.0 software using a log(inhibitor) versus normalized response. Experiments were performed in triplicates on different days using independently grown cell cultures.

\section{i. Translocation across a human endothelial cell line}

The in vitro translocation capacity of antibody fragments was evaluated using an in vitro HBEC-5i cell model, as previously described. Briefly, HBEC-5i cells were carefully harvested with trypsin-EDTA and seeded 8000 cells/well in pre-coated tissue culture inserts (transparent polyester (PET) membrane with $1.0 \mu \mathrm{m}$ pores) for 24-well plates (BD Falcon, New York, USA). During 8 days, medium was changed every other day. After medium removal, cells were washed two times with $1 x$ PBS and one time with DMEM:F12 medium without phenol red. Then, $25 \mathrm{ng}$ of previously diluted anti-body fragments were added to the apical side of the in vitro BBB model and incubated for 6 and 24h. Then, samples were collected from the apical and basolateral side and loaded onto $12 \%$ acrylamide Bis-Tris gels and run in Tris/Glycine/SDS running buffer (25 mM Tris, $192 \mathrm{mM}$ Glycine, 0.1\% SDS, pH 8.3). The resolved antibody fragments were transferred from the gel onto a nitrocellulose membrane in Tris/Glycine transfer buffer (25 mM Tris, $192 \mathrm{mM}$ Glycine, 20\% (v/v) methanol, pH 8.5) at $250 \mathrm{~mA}$ for $90 \mathrm{~min}$. Membranes were blocked with $5 \%$ low fat milk in 1x PBST, then blotted with a HRP-preadsorbed anti-human IgG antibody (1:10,000, in 1x PBS). The blots were revealed using ECL Prime Western Blotting Detection Reagent according to the manufacturer's instructions. Then, imaged using the Amersham Imager 600 (GE Healthcare; Chicago, Illinois, USA). Experiments were performed on different days using independently grown cell cultures.

\section{j. In vitro BBB model integrity assay}

In the end of the translocation assay, an in vitro BBB integrity assay was performed. Herein, cells were washed two times with 1x PBS and one time with DMEM:F12 medium without phenol red. Then, previously diluted FD40 was added to the apical side and incubated for $2 \mathrm{~h}$. FD40 was diluted in DMEM:F12 medium without phenol red to an absorbance below 0.1. Finally, samples from the apical and basolateral side were collected and fluorescence intensity analyzed using a Varioskan ${ }^{\mathrm{TM}}$ LUX multimode microplate reader. The percentage of FD40 recovered was determined using the following equation:

FD40 Permeability $(\%)=\left(\frac{F_{i}-F_{\text {cells }}}{F_{F D 40}-F_{\text {Medium }}}\right) \times 100$

Eq. 2 


\section{SUPPORTING INFORMATION}

$F_{i}$ is the fluorescence intensity recovered, $F_{\text {cells }}$ is the fluorescence intensity recovered from cells without treatment, $\mathrm{F}_{\mathrm{FD} 40}$ is the fluorescence intensity of total FD40 initially added to the transwell apical side, and $\mathrm{F}_{\text {Medium }}$ is the fluorescence intensity of the medium.

The integrity of the in vitro BBB model is indirectly proportional to the percentage of FD40 recovered and was determined using the following equation:

$$
\text { Integrity }(\%)=100-F D 40 \text { Permeability }(\%)
$$

Eq. 3

\section{k. FcRn binding affinity}

96-well polystyrene ELISA plates (Corning, USA) were coated with $2 \mu \mathrm{g} / \mathrm{mL}$ (100 $\mu \mathrm{L} /$ well) FcRn (R\&D Systems; Minneapolis, USA) diluted in $100 \mathrm{mM} \mathrm{NaPO4}$, pH 6.0, 0.05\% Tween 20 (v/v), and 0.1\% BSA $(\mathrm{m} / \mathrm{v})$ at $4^{\circ} \mathrm{C}$ o.n. The coated wells were then incubated with a blocking solution $(0.5 \% \mathrm{BSA})$ for $2 \mathrm{~h}$ at r.t., washed 4 times and incubated for $1 \mathrm{~h}$ with serially diluted antibody fragments at r.t. $(0.1-$ $10,000 \mathrm{nM}$ range). After 4 more washes, HRP-conjugated goat anti-polyhistidine antibody $(1: 10,000)$ was added to the plates for protein detection. After another 4 washed, the plate was developed by adding $50 \mu \mathrm{L}$ of Ultra-TMB substrate to each well. The absorbance was measured at $450 \mathrm{~nm}$ using a VarioskanTM LUX multimode plate reader. The binding data were GraphPad Prism 7.0 software using a single-site kinetic fit model. Experiments were performed in triplicates.

\section{Statistical analysis}

Quantitative data were processed using Excel 2013 (Mi-crosoft; New York, USA) and the GraphPad Prism 7.0 soft-ware package. Medians, means and standard deviations are shown in the figures and tables. Pairwise significances were calculated using one-way ANOVA followed by Tukey's multiple comparison test, nonparametric Mann-Whitney, Kruskal-Wallis, and two-way ANOVA by Sidak's multiple comparison test. 


\section{SUPPORTING INFORMATION}

\section{Results}
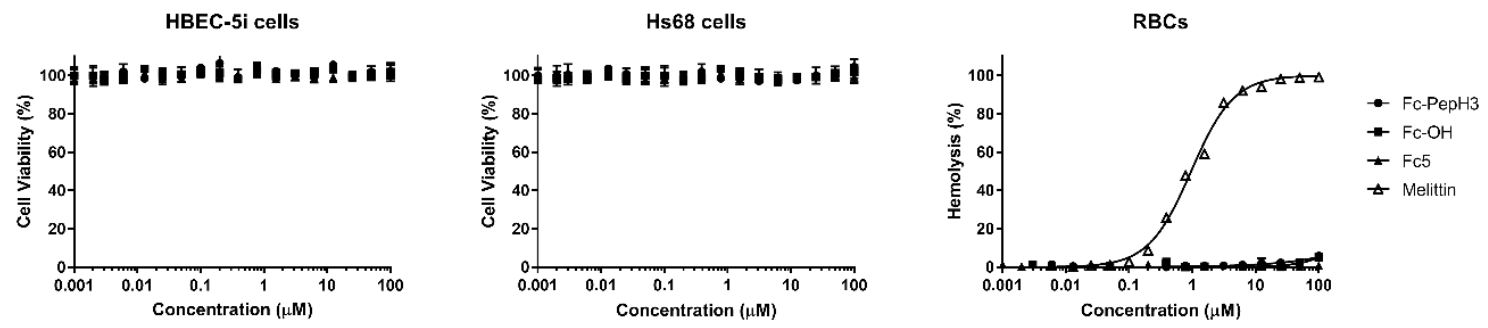

Figure S2. in vitro toxicity of antibody fragments. HBEC- 5 , $\mathrm{Hs} 68$, and RBCs were incubated with $100 \mu \mathrm{L}$ of previously diluted antibody fragments $(0.001-100.0 \mu \mathrm{M}$ range) for $24 \mathrm{~h}$. The values were obtained from triplicates of three independent experiments. Error bars, S.D. 


\section{SUPPORTING INFORMATION}

Fc-PepH3

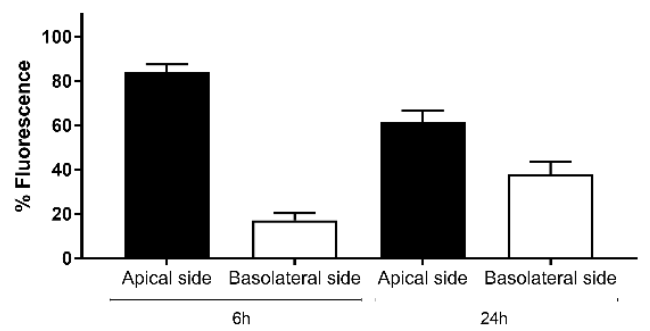

FC5

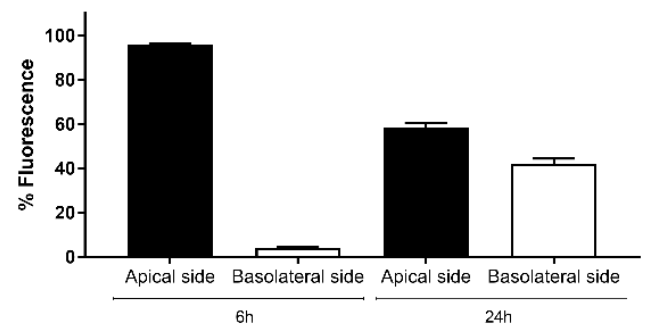

$\mathrm{Fc}-\mathrm{OH}$

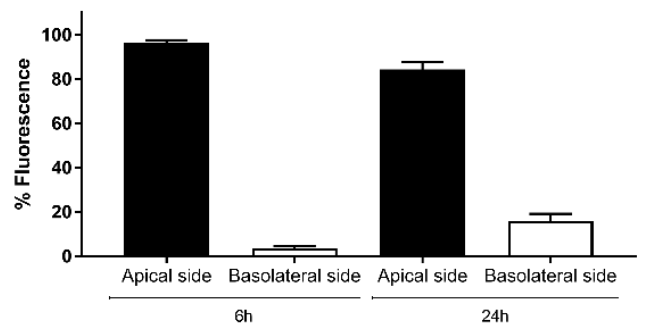

BBB Permeability - FD40

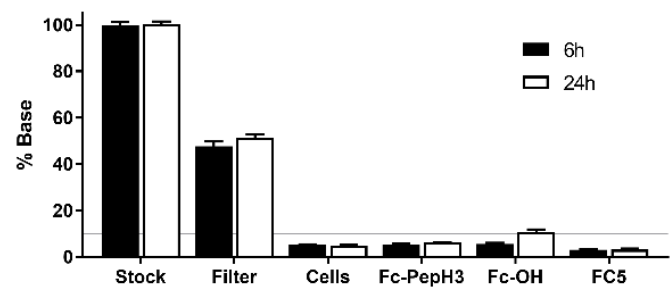

Figure S3. Translocation of antibody fragments across an in vitro BBB model and the FD40 permeability study. Percentage of translocation of antibody fragments ( $25 \mathrm{ng}$ ) and fluorescence intensity of FD40 measured after translocation assay. The values were obtained from triplicates of three independent experiments. Error bars, S.D. 


\section{SUPPORTING INFORMATION}

\section{FcRn Binding Affinity}

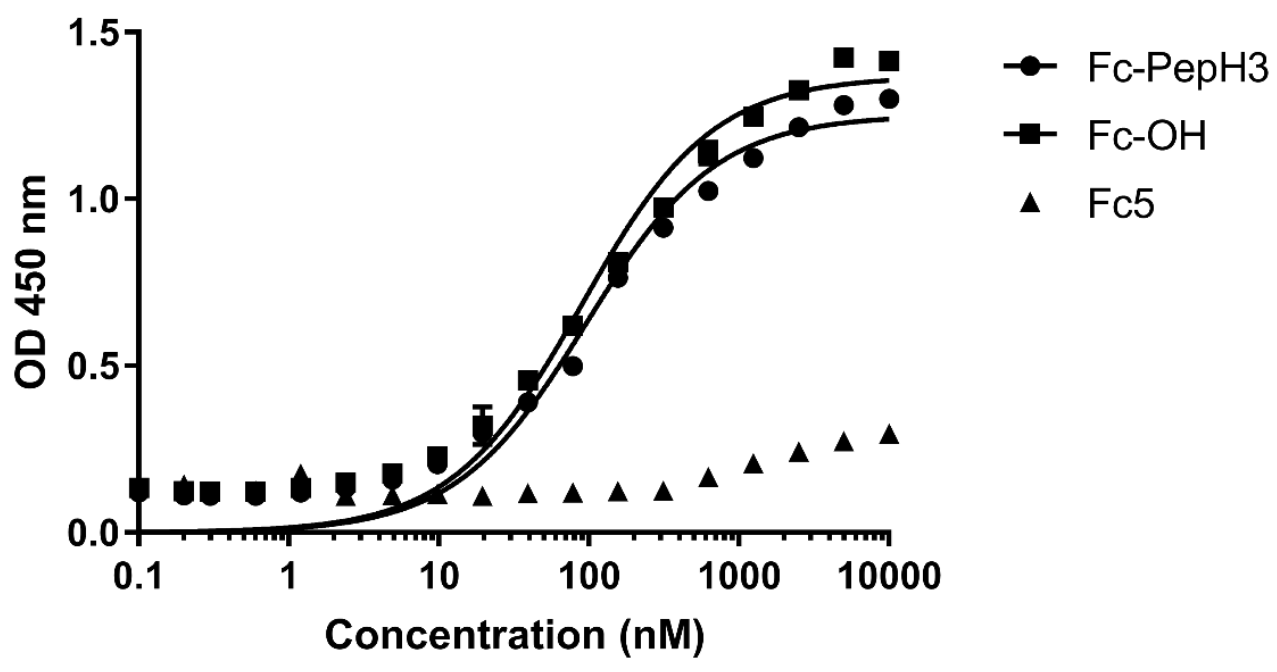

Figure S4. Binding of proteins to the FcRn. Antibody fragments' binding curves to immobilized FcRn at pH 6.0, obtained using a single-site fit model. The values were obtained from triplicates of three different experiments. Error bars, S.D. 


\section{SUPPORTING INFORMATION}

\section{Supplementary data - in vitro HBEC-5i characterization}
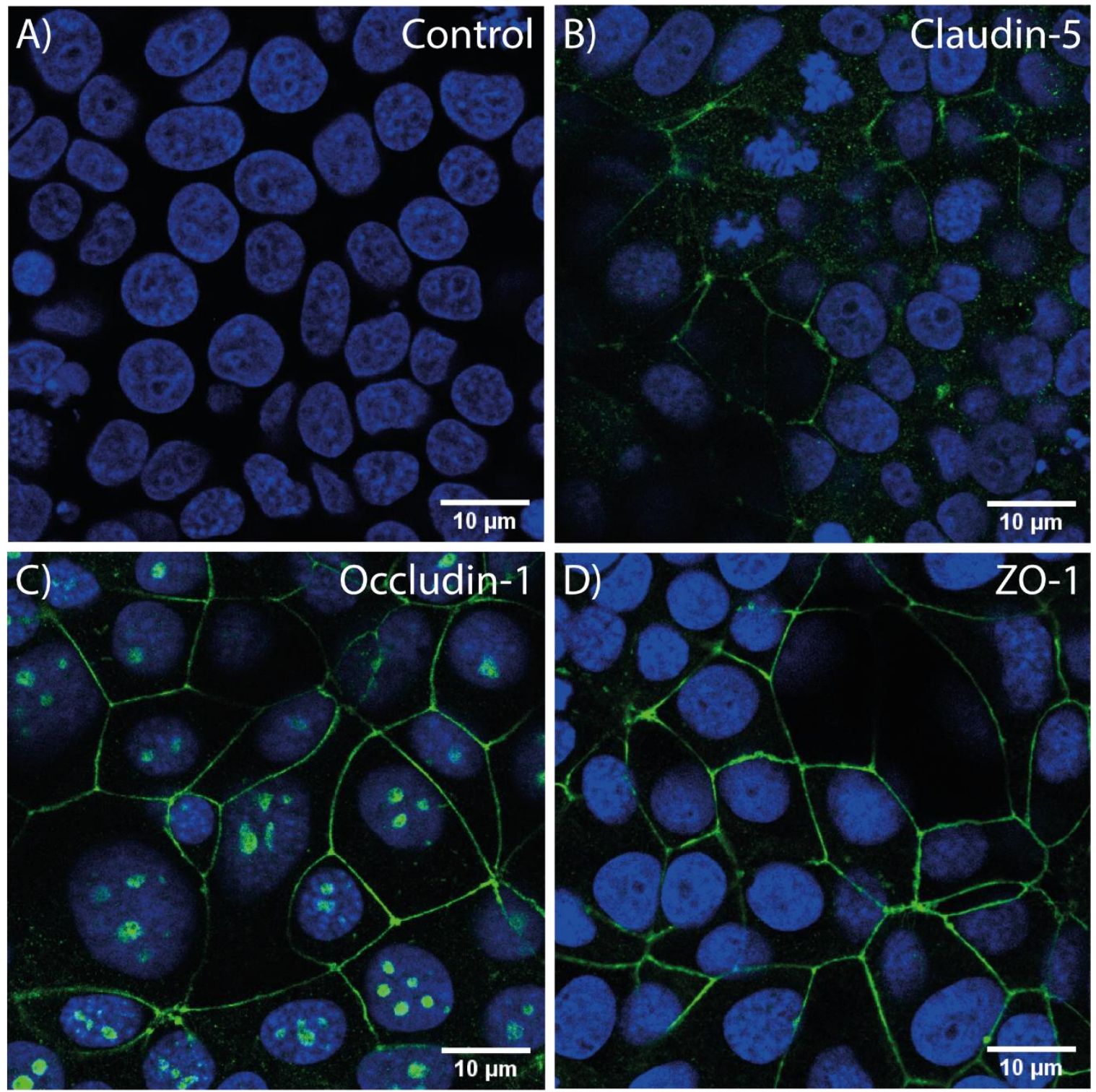

Figure S5. Confocal images of tight junction proteins in HBEC-5i cells. (A) Control, (B) Claudin-5, (C) Occludin-1, and (D) ZO-1. Images were obtained using a 40x water-objective. Nuclei stained with Hoeschst 33342 (blue) and tight junction proteins (red). Bar represents $10 \mu \mathrm{m}$. 


\section{SUPPORTING INFORMATION}

\section{Transport of LY across HBEC-5i monolayer}

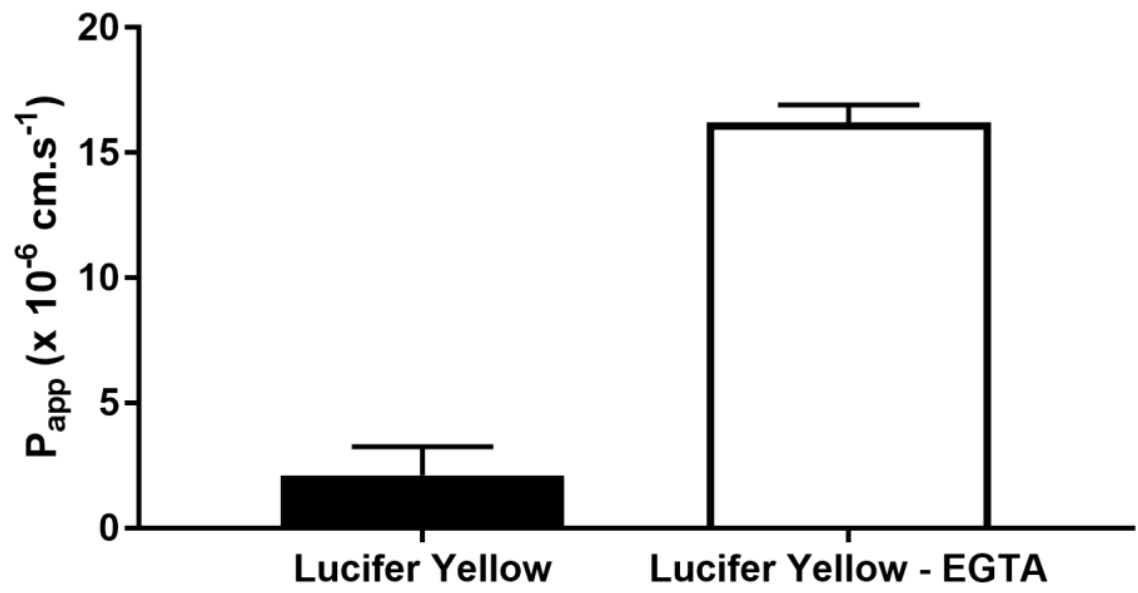

Figure S6. Transport of Lucifer Yellow (LY) across HBEC-5i monolayers. Ly applied at a concentration of $50.0 \mu \mathrm{M}$ in serum-free medium. HBEC-5i monolayers were incubated with LY in or without the presence of EGTA. Experiments were performed in triplicates on different days using independently grown cell cultures. Data is shown as mean \pm SD. 


\section{SUPPORTING INFORMATION}

\section{Transport of hTf across HBEC-5i monolayer}

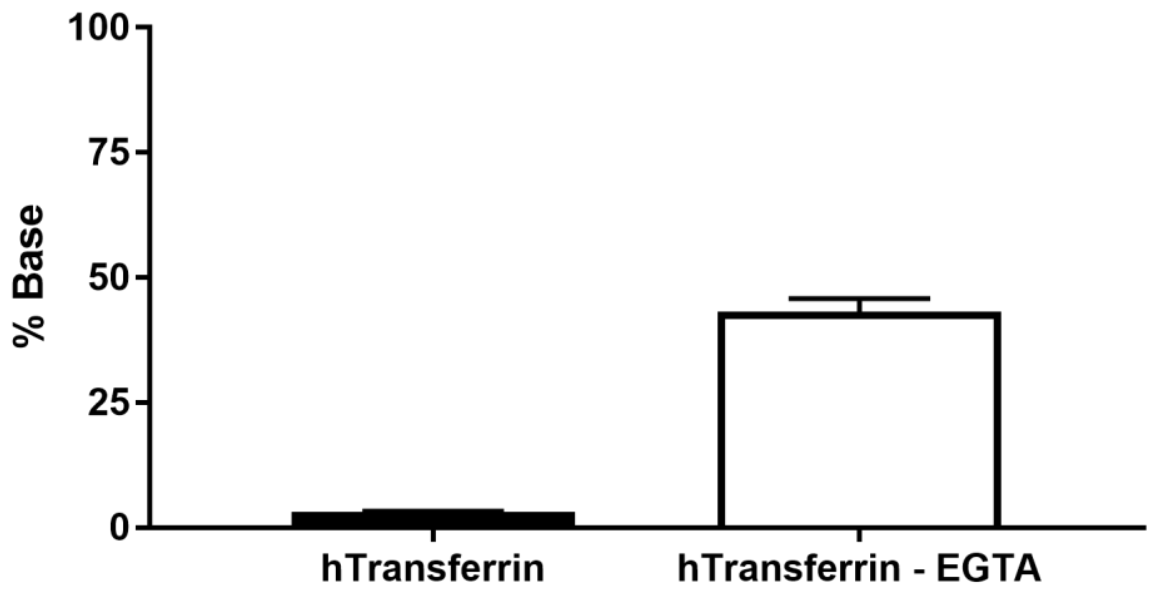

Figure S7. Transport of human Transferrin (hTf) across HBEC-5i monolayers. hTf applied at a concentration of 400.0 $\mu \mathrm{g} / \mathrm{mL}$ in serum-free medium. HBEC-5i monolayers were incubated with $\mathrm{hTf}(24 \mathrm{~h})$ in or without the presence of EGTA. Experiments were performed in triplicates on different days using independently grown cell cultures. Data is shown as mean $\pm S D$ 


\section{SUPPORTING INFORMATION}
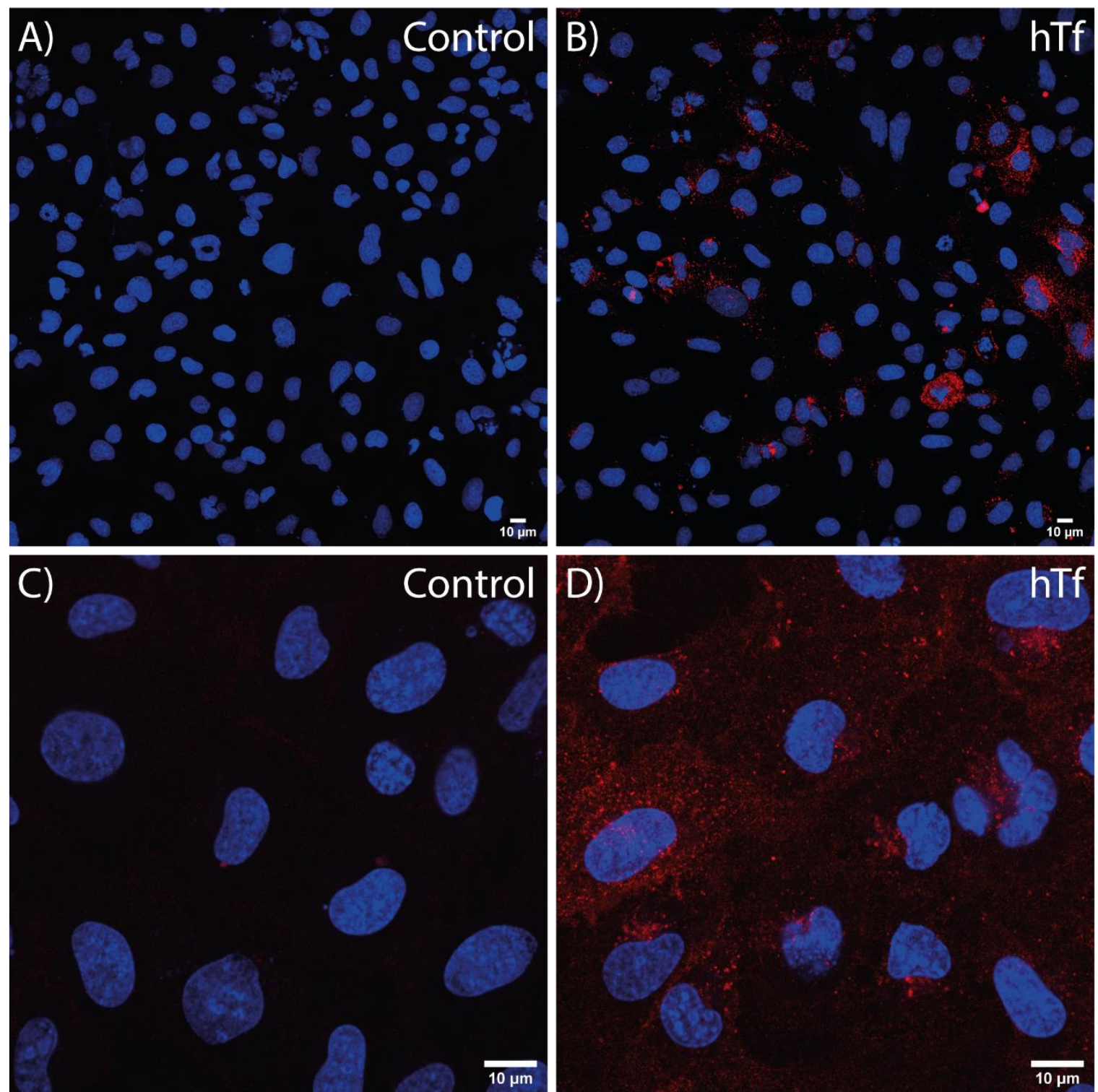

Figure S8. Confocal images of HBEC-5i cells incubated for $20 \mathrm{~min}$ at $37^{\circ} \mathrm{C}$ with Human Transferrin at a concentration of $25 \mu \mathrm{g} / \mathrm{mL}$ in HEPES supplemented with $1 \mathrm{M}$ glucose, $0.2 \mathrm{M} \mathrm{MgCl}_{2}$, and 1\% BSA. (A - B) 20x air-objective, (C - D) 63x oilobjective. Nuclei stained with Hoeschst 33342 (blue) and transferrin Alexa Fluor 568 conjugate (red). Bar represents 10 $\mu \mathrm{m}$. 